total curative effect of the observation group was significantly higher than that of the control group, and the incidence of adverse reactions in the observation group was also less than that in the control group. Its indicate that the treatment has a significant effect of Zhibai Dihuang Pill on senile vaginitis.

2 periodontitis and gingivitis Luo Xia [2] [3] respectively with randomly selected chronic periodontitis patients as the research object, divided into two groups, the control group with routine periodontal treatment, the experimental group used zhibodihuang treatment, analysis of two groups of treatment effect. two groups is significant difference $(\mathrm{P}<0.05)$ after treatment, compared with of periodontal indexes, IL-6, IL-1, TNF- $\alpha, C$ reactive protein and total clinical efficiency, The experimental results show zhibaidihuang pill combined with periodontal therapy has good curative effect on redution chronic periodontitis inflammatory factors, not only enhance the treatment effect, effectively reduce the inflammatory factor can promote the repair and regeneration of periodontal tissues. Huang Jianyu [4] selected recurrent episodes of acute gingivitis patients as the research object, observated the inflammation level before and after treatment. The results show that Zhibaidihuang pill combined with antibiotics can treat acute gingivitis.

3 nephritis Zhibaidihuang decoction treat the patients with acute pyelonephritis, the control group was given antibiotic treatment, the treatment group was given oral eight powder and Zhibaidihuang pill . The positive incidence of urine bacteria culture, the main symptoms and signs disappeared time of patients in the treatment group were better than those in the control group [5], yung-Ho Hsu[6] proved that zhibaidihuangwan has a protective effect on apoptosis of rat kidney cells, which happened by gentamicin

4 male reproductive inflammatory diseases Donghua [7] reviewed the clinical situation, the research progress and the treatment mechanism about object for each type of male infertility,. Liu Yongxin [8] reported Zhibaidihuang decoction combined with massage therapy has significant curative effect for spermatorrhea.

Wu Jinping [9] proved that Zhibaidihuang pill combined erzhi pill has good curative effect on chronic Vesiculitis. The control group was given roxithromycin + Pudilanxiaoyan tablets treatment; the observation group was given with Zhibaidihuang pill combined erzhi pill treatment, the observation group cure time was significantly shorter than the control group, and semen volume and sperm motility index in observe group increased more significantly than the control group.

In conclusion, Zhibaidihuang pill have curative effect on anti-inflammatory effect. In the study, the research methods was devolopment from the treatment rate of simple summary expanded to statistical analysis, the index from the macro effect of in-depth observation into the cell or the microscopic factor, it is provide basis for clinical application and modern pharmacological study of Zhibaidihuang pill.

\title{
Reference
}

1 Ruan Senlian, Asia, Meng Xiangling. Zhibaidihuang pill in the treatment of senile vaginitis clinical curative effect analysis of // and clinical pharmacology of traditional Chinese medicine. - 2015 (01) - C.302

2 Luo Lihong. Zhibaidihuang pill for chronic periodontal maintenance therapy the clinical curative effect of // in northern medicine- $2016(04)$ - C. 82-83.

3 Xia. Chengjian Zhibaidihuang Decoction on chronic periodontitis inflammatory factors // and clinical pharmacology of traditional Chinese medicine-2015 (01) - C.277-278.

4 Huang Jianyu. Zhibaidihuang pill in the treatment of 12 cases of acute gingivitis // Guangdong occupation technology education and research- 2016 (04) - C.194-196.

5 Yang Lin, Wang Rong. Eight is scattered and Zhibaidihuang pill in the treatment of 60 cases of acute pyelonephritis with // Chinese TCM modern distance education-2014 (09) - C.132-133.

6 Yung-HoHsu, Tso-Hsiao Chen, Mei-Yi Wu, et al. Protective effects of Zhibai Dihuang Wan on renal tubular cells affected with gentamicin-induced apoptosis // Journal of Ethnopharmacology. -2014-151(2014) - C.635-642.

7 Han Zhong, Donghua, where the application of Qing Hu zhibaidihuangwan in male infertility //.China science, -2016 (12) C.84-87.

8 Liu Yongxin, Yang Chunmei. Zhibaidihuang decoction combined with massage therapy efficacy // Chinese spermatorrhea community physicians-2016(17) - C.101+103.

9 Wu Jinping, Zheng Jianlong, Wei Mingquan, Liu Yannan. Two to zhibaidihuangwan combined pill in the treatment of chronic Vesiculitis curative effect observation // new Chinese medicine- 2015(12) - C.84-86.

\section{DOI 10.22448/AMJ.2017.3.54-55 \\ CLINICAL OBSERVATION ON THE TREATMENT OF MILD COGNITIVE IMPAIRMENT BY COMBINED ACUPUNCTURE AND MEDICATION}

\author{
PangJing \\ The Second Affiliated Hospital of Heilongjiang TCM University, 150001, Harbin, China ; EMAIL : XKP850713@126. \\ COM
}

Abstract : To observe the therapeutic effect of acupuncture plus medication in the treatment of mild cognitive impairment of liver-kidney insufficiency type.

Key words:Acupuncture;Acupuncture medication combined;Mild cognitive impairment; donepezil

54 Амурский медицинский журнал №3 (19) 2017 
Objective:To observe the therapeutic effect of acupuncture plus medication in the treatment of mild cognitive impairment of liver-kidney insufficiency type.

Method:Sixtysubjects diagnosed with mild cognitive impairment of liver-kidneyinsufficiencytype were randomlyassigned to an acupuncture group, an acupuncture-medication group and a medication group.The acupuncture group used Baihui,Shenting,Benshendual sides,Ganshudual sides, Shenshudual sides,Neiguandual sides,Hegudual sides,Zhaohaidual sidesand Taichongdualsides.Theacupuncture-medicationgroupusedanlishen(Donepezil) basisintheacupuncturegroup. The medication group adapt anlishen(Donepezil).Each group was treated for 28 days.3.The Mini-Mental State Examination (MMSE),Montreal Cognitive Assessment (MoCA), as well as the measurement of P300 latency and amplitude were adopted in the evaluation before and after treatment. 4.All data was processed bySPSS17.0 statistical software on the computer.

Result:Before and after treatment,in comparison of MMSE,MoCA,P300 latency and amplitude,the results in the acupuncture group,the acupuncture-medication group,the medication group were statistically significant $(\mathrm{P}<0.05)$.In comparison of MMSE,MoCA, P300 latency and amplitude, the results in the acupuncture-medication group were significantly different from that in the acupuncture group and medication group $(P<0.05)$; but the differences between the acupuncture group and medication group were not statistically significant $(P>0.05)$.

Conclusion: 1 ..Three types of treatment on mild cognitive impairment of liver-kidney insufficiency type are valid.

2.Among the three solutions, acupuncture plus medication is the best in the treatment of mild cognitive impairment of liver-kidney insufficiency type.

References:

[1]Helge Horn,Nadeem Syed,Heinrich Lanfermann,et al.Cerebral networks linked to the event-related potential P300[J].Eur Arch Psychiatry Clin Neurosci,2003,253:154-9

[2]Goodin DS,Squires KC,Starr A,et al.Long latency event-related components of auditory evoked potential in dementia[J].Brain 1978,101:635-48

[3]Saito H,Yamazaki H,Matsuoka H,et al.Visual event-related potential in mild dementia of the Alzheimer's type[J].Psychiatry Clin Neurosci,2001,55(4):365-71

[4]P.Stanzione,R.Semprini,M.Pierantozzi,et a1.Age and stage dependency of P300 latency alterations in non-demented Parkinson's disease patients without therapy. Electroencephalography and Clinical[J].Neurophysiology,1998,108(1):80-9|

[5]Sumi N,Nan'no H,Fujimoto O,et al.Interpeak latency of auditory event-related potentials(P300) in senile depression and dementia of the Alzheimer type[J].Psychiatry Clin Neurosci,2000,54:679-84

UDC 616.127-08-035 DOI 10.22448/AMJ.2017.3.55-56

EFFECTIVENESS OF CITOFLAVIN IN PATIENTS WITH ACUTE MYOCARDIAL INFARCTION

\section{D.I. Pereverzev, N.V. Simonova, V.A. Dorovskikh, A.I. Sushkova, L.P. Zueva, E.A. Nesterenko, L.I. Demidenko}

\section{Amur State Medical Academy, Blagoveshchensk, Russia}

Summary. To study the effect of Cytoflavin on parameters of systolic left ventricular function in acute myocardial infarction. 46 patients with standard therapy received the drug Cytoflavin (Polysan, St.Petersburg, Russia) intravenously $20 \mathrm{ml}$ of the solution diluted in $250 \mathrm{ml} 5 \%$ glucose solution, 1 times a day 30 minutes before coronary artery recanalization; 60 patients (control group) received only standard therapy. The drug's effectiveness was evaluated in terms of systolic left ventricular function: ejection fraction, end-diastolic volume, end-systolic volume. The inclusion of Cytoflavin in the treatment of patients with acute myocardial infarction should be considered as pathogenetically justified clinicallyjustified and promising.

Key words: cytoflavin, acute myocardial infarction, systolic left ventricular function, ejection fraction, troponin I, patients.

Myocardial ischemic injury, currently occupying a leading position in the rank of priority problems of cardiology, is one of the most frequent causes of myocardial infarction, which occurs circulatory disorders are crucial for the disease and for the nearest and remote forecast of life of patients with a high early morbidity and mortality. In addition to ischemic myocardial damage, severity of acute myocardial infarction is determined by the degree of metabolic disorders caused by the reduction of the heart. On the one hand, the depth of metabolic disorders caused by a primary lesion of the myocardium and are associated with these disorders of the Central hemodynamics, hypoxia and the body's reaction to damage to the heart muscle; on the other hand, secondary metabolic disorders could exacerbate violations of the contractility of the heart and to influence the outcome of the disease. This leads to the desirability of finding means of pharmacological correction of hypoxic and metabolic disorders, to their use in the early stages of myocardial ischemia.

In recent years, in complex therapy of myocardial ischemia include combination drug Cytoflavin developed "POLYSAN" (Saint-Petersburg, Russia), which is composed of riboxin, riboflavin, nicotinamide, succinic acid. Despite a few articles that reflect the results of clinical trials succinaldehyde of the drug in complex therapy of myocardial infarction, searching for new evidence of the efficacy of Cytoflavin as an adjuvant pharmacological component of reperfusion therapy is in our view a promising and reasonable. 